\title{
Active avoidance conditioning with dorsal central gray stimulation in a place preference paradigm
}

\author{
VICKI J. ROBERTS and VERNE C. COX \\ University of Texas at Arlington, Arlington, Texas
}

\begin{abstract}
Avoidance conditioning in response to aversive dorsal central gray stimulation was examined in a simple place preference task that minimized response demands and provided highly salient environmental cues. In contrast to shuttlebox avoidance learning with central gray stimulation, a high incidence of active avoidance learning was observed. The results suggest that poor avoidance in a shuttlebox task with central gray stimulation reflects task complexity rather than a fundamental inability to associate environmental cues with the consequences of central gray stimulation.
\end{abstract}

Considerable evidence exists to suggest a role for the mesencephalic central gray in the transmission of nociceptive sensory input. Results from behavioral studies have demonstrated that electrical stimulation of this region is aversive (Cox, 1967; Cox \& Valenstein, 1966; Olds \& Olds, 1962; Stein, 1965; Valenstein, 1965) and that lesions of this area decrease responsiveness to nociceptive peripheral stimulation (Liebman, Mayer, \& Liebeskind, 1970; Melzack, Stotler, \& Livingston, 1958). In addition, sensations evoked by stimulation of the central gray in humans have been described as painful or unpleasant (Nashold, Wilson, \& Slaughter, 1969). Medially coursing fibers of the spinothalamic tract, which convey pain information in humans and nociceptive input in animals, terminate in the central gray region (Mehler, 1957). Although this evidence implicates the central gray in supraspinal transmission of nociceptive input, less is known with regard to the functional properties of this input and its relationship to other neural systems that respond to nociceptive stimuli.

Previous work has indicated that central gray stimulation will yield efficient escape and passive avoidance responding (Cox, 1967). However, two-way shuttlebox active avoidance learning in response to central gray stimulation is inferior to that obtained with aversive peripheral stimulation (Cox, 1967). This avoidance deficit cannot be accounted for in terms of a memorial deficit because animals are able to master a passive avoidance task with central gray stimulation. In addition, freezing behavior cannot account for the shuttlebox deficit because d-amphetamine sulphate (Krieckhaus, Miller, \& Zimmerman, 1965) does not improve task performance (Cox, 1967). Irisawa and Iwasaki (1986) also reported a deficit in active avoidance conditioning with cental gray stimulation. They speculated that central gray stimulation will not support avoidance learning because such stimulation

Send reprint requests to Vicki J. Roberts, University of Texas at Arlington, Department of Psychology, Box 19528, Arlington, TX 76019. engages motor systems rather than nociceptive systems. However, successful passive avoidance behavior would appear to argue against this contention.

Other investigators have suggested that the failure to obtain avoidance learning with central gray stimulation may reflect an initial rewarding component associated with the onset of stimulation. Wolfle, Mayer, Carder, and Liebeskind (1971) reported that rats will escape but not avoid central gray stimulation that yields self-stimulation behavior. However, self-stimulation behavior has been observed only in ventral sites, whereas deficient avoidance learning has been observed at both ventral and dorsal central gray sites.

The present study was designed to examine active avoidance learning in response to dorsal central gray stimulation. It is possible that the active avoidance deficits observed with aversive dorsal central gray stimulation reflect task complexity, particularly in the case of the shuttlebox task (Cox, 1967). Alternatively, a fundamental inability to associate the consequences of dorsal central gray stimulation with environmental cues might account for the active avoidance deficit. In order to investigate these alternatives, we employed a simple place preference task that minimized response demands and provided highly salient environmental cues that differentiated "safe" and "unsafe" locations. Successful avoidance would indicate that central gray stimulation can support associative learning involving environmental cues and the consequences of aversive central gray stimulation. Experiment $1 \mathrm{em}-$ ployed footshock to assess the utility of the place preference task for investigation of avoidance learning. Experiment 2 employed central gray stimulation in the same testing situation.

\section{EXPERIMENT 1}

\section{Method}

Subjects. Experiment 1 employed 24 Holtzman rats (females, 250-350 g; males, $450-500 \mathrm{~g}$ ). They were individually housed and 
maintained on a 12-h/12-h light/dark cycle with ad-lib food and water.

Apparatus. The conditioned place preference task (Phillips, Spyraki, \& Fibiger, 1982) employed a rectangular wooden chamber $156 \times 26 \times 43 \mathrm{~cm}$ with a shock grid floor. The inside of the chamber, black on one half and white on the other, could be partitioned into two separate compartments by lowering a clear sliding door. Two photocells, located $16.5 \mathrm{~cm}$ from the end of each compartment, provided information regarding the position of the animal in the chamber.

Procedure. Three groups of subjects were used. Individual subjects were initially placed on the white side of the chamber on the evening prior to testing. Water was placed in the center of the chamber and food pellets were equally distributed on each side. At 10:30 a.m. the following day, food and water were removed and the animal was placed in the white compartment of the chamber. After 5 min of habituation, side preference was measured by recording time spent on each side of the chamber for the next $1,800 \mathrm{sec}$. Animals were then confined by the sliding partition within the preferred side for $800 \mathrm{sec}$

Group 1. Group 1 consisted of 10 female rats. During the last $200 \mathrm{sec}$ of confinement in the preferred side of the chamber, the animals were administered five 0.5 -mA shocks $0.5 \mathrm{sec}$ in duration every $\mathbf{4 0} \mathrm{sec}$ via a shock scrambler. After the last shock, each animal was removed for $10 \mathrm{~min}$ and placed in the home cage. During this period, the sliding partition was removed, fresh bedding paper was placed beneath the chamber, and the grid floor and chamber were cleaned to minimize olfactory cues. The animals were then returned to the initially preferred compartment for the retest trial and an 1,800 -sec sample of side preference was obtained.

Group 2. Group 2 consisted of 5 female rats. They were treated in the same manner as animals in Group 1 except that they were placed on the nonpreferred side of the chamber on the retest trials.

Group 3. Group 3 consisted of 6 male and 3 female rats that had been implanted with intracranial electrodes. They were employed to assess the effects of confinement and electrode cable attachment on side preference. They were treated the same as animals in Group 1 except that they received no aversive stimulation during testing and were attached to an electrode cable $5 \mathrm{~min}$ prior to initial preference measurement.

\section{Results}

Group 1. The subjects in Group 1 were placed in the initially preferred side of the chamber after receiving footshock. Successful avoidance behavior required active movement from the preferred compartment to the nonpreferred compartment and was reflected by a shift in compartment preference. All 10 animals moved into the nonpreferred compartment and there was a significant decrease in amount of time spent on the initially preferred side on the retest trial $[\mathrm{t}(9)=11.62, p<.01]$.

Group 2. To ensure that the avoidance behavior displayed by Group 1 animals did not reflect a nondirectional response, animals in Group 2 were placed on the initially nonpreferred side of the chamber for the retest trial. There was a significant decrease in amount of time spent on the initially preferred side $[t(4)=10.44, p<.01]$. A repeated measures ANOVA yielded no significant difference between Groups 1 and 2 on either the pretest measure $[F(1,13)=1.74, p>.05]$ or the posttest measure $[F(1,13)=.58, p>.05]$.

Group 3. Group 3 was employed to assess the effects of confinement and electrode cable attachment in order to assure that these variables would not produce avoidance behavior independently of aversive stimulation. Confinement and electrode cable attachment had no effect on postconfinement side preference $[t(8)=.63, p>.05]$.

Overall, the results of Experiment 1 demonstrated that directional active avoidance and side preference shifts can be easily obtained in a simple place preference task with conventional footshock. In addition, confinement combined with electrode cable attachment had no measurable effects. These findings established the utility of the task for examining the effects of central gray stimulation on active avoidance and side preference in Experiment 2.

\section{EXPERIMENT 2}

\section{Method}

Surgery. Ten female and 10 male Holtzman albino rats were prepared with bipolar electrodes (Plastic Products) implanted in the area of the dorsal central gray and overlying tegmentum. With the skull level between bregma and lambda, electrodes were implanted at a lateral angle of $25.5^{\circ}, 2.5 \mathrm{~mm}$ lateral to the midline, $7.0 \mathrm{~mm}$ posterior to bregma, and 4.7 to $4.9 \mathrm{~mm}$ below the skull surface. At the conclusion of the experiment, the animals were sacrificed with an overdose of anesthetic and perfused intracardially with a $0.9 \%$ solution of saline followed by a $10 \%$ solution of formalin. The brains were removed, frozen, and sliced in 80-M sections and stained with thionin.

Stimulation parameters and behavioral ratings. Brain stimulation consisted of 0.5 -sec trains of $100 / \mathrm{sec}$ pairs of biphasic square pulses of 0.2 msec duration delivered by a pair of Grass S9 stimulators. A 4-point behavioral rating scale was used to equate the behavioral reactions of animals receiving $0.5 \mathrm{sec}$ trains of aversive brain stimulation with the behavioral responses of animals that were administered $0.5 \mathrm{~mA}$ footshocks in Experiment 1. Thus individual brain stimulation parameters reflected the lowest current intensity that evoked vigorous but controlled escape-like responses that closely resembled the responses observed in animals administered footshocks. Currents used ranged from $95 \mu \mathrm{A}$ to $400 \mu \mathrm{A}$.

Procedure. The apparatus, pretest, and posttest procedures were the same as those applied to Group 1 in Experiment 1. The optimal stimulation current was established for each animal as quickly as possible during the initial portion of the 800 -sec period of confinement within the preferred side of the chamber. During the last $200 \mathrm{sec}$ of confinement, a total of five 0.5 -sec trains of brain stimulation were administered at the rate of one train every $40 \mathrm{sec}$. Because of the need to establish the appropriate current level during the initial portion of the confinement period, the total number of aversive stimuli delivered varied across subjects and was greater than the fixed number of five footshocks delivered to animals in Experiment 1. However, the primary purpose of this study was not to directly compare avoidance behavior in response to footshock and central gray stimulation, but rather to determine whether a high incidence of avoidance behavior could be obtained with central gray stimulation in a behavioral task that yielded efficient avoidance responding with a footshock.

\section{Results}

A repeated measures ANOVA yielded a significant shift in place preference after the presentation of aversive central gray stimulation $[F(1,18)=50.25, p<.01]$. No significant main effect for sex $[F(1,18)=.92, p>.05]$ or significant sex $\times$ preference shift interaction $[F(1,18)=$ $.30, p>.05]$ was observed. There was no significant correlation between pretest and posttest preference scores 


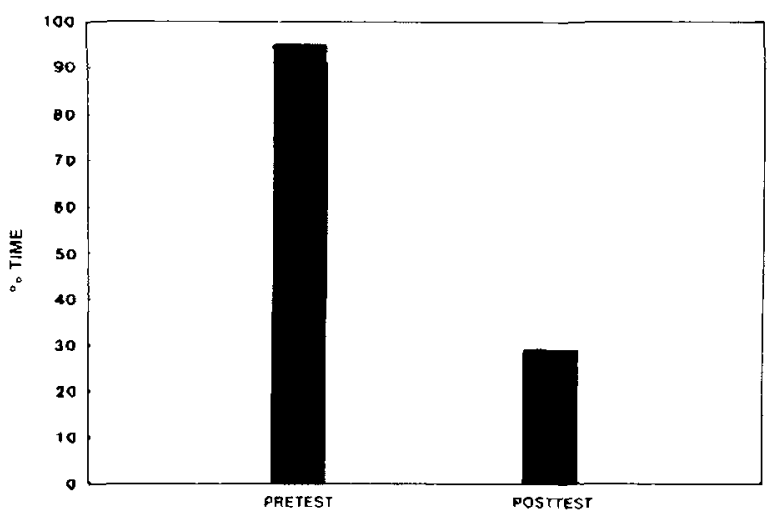

Figure 1. Percentage of time spent in preferred compartment in an 1,800-sec test prior to and after exposure to aversive midbrain stimulation.

$(r=.19, p>.40)$. As can be seen in Figure 1, the percentage of time spent in the initially preferred side decreased over all subjects from a mean of $95 \%$ to $29 \%$.

Only 5 of the 20 animals displayed poor avoidance of the compartment associated with central gray stimulation on the retest trial. Of these 5 animals, only 2 failed completely to avoid the compartment associated with the aversive brain stimulation. This was evidenced by complete failure to cross into the initially nonpreferred compartment.

\section{Histological Analysis}

The placements of all electrode tips of subjects employed in the present study were within the dorsomedial tegmentum extending from the deeper layers of the superior colliculus to 0.1 to $0.2 \mathrm{~mm}$ within the border of the most dorsal aspect of the central gray. All placements were within or near the terminal fields of the spinothalamic fiber tract described by Mehler (1957).

A systematic examination of the anatomical loci of the electrodes was performed to determine whether variations in either current intensity or avoidance behavior were related to electrode placement. The area encompassing the electrode sites was partitioned into quadrants. The mean current intensities for each quadrant were $244 \mu \mathrm{A}$, dorsal-medial $(n=5) ; 293 \mu \mathrm{A}$, dorsal-lateral $(n=8)$; $181 \mu \mathrm{A}$, ventral medial $(n=4)$; and $186 \mu \mathrm{A}$, ventral-lateral $(n=3)$. Three a priori contrasts revealed that a significantly higher current intensity was required with dorsal than with ventral placements $[F(1,16)=4.99, p<.05]$ and with dorsal-lateral placements relative to other sites $[F(1,16)=5.94, p<.05]$. No differences in current intensities were found between medial and lateral placements $[F(1,16)=.52, p>.05]$. These results indicate that electrode sites further away from spinothalamic terminal fields require higher current intensities to yield behavioral reactions comparable to more proximal sites.

No relationship was expected between electrode site and avoidance behavior, because current parameters were ad- justed to yield similar behavioral reactions across sites. The same anatomical contrasts as above confirmed this expectation. Nor was there a significant correlation between current intensity and percentage change scores for side preference $(r=.28, p>.22)$. It should be noted that this is also a reflection of the relationship between percentage change score and total number of stimulations received, because higher terminal current intensities required more ascending exploratory stimulations. In sum, these results indicate that activation of the spinothalamic fiber terminal fields was the critical factor in evoking aversive reactions.

\section{DISCUSSION}

The present study demonstrated that rats can acquire a simple active avoidance response with dorsal central gray stimulation. Avoidance behavior was evidenced both by active movement away from stimuli that were associated with the presentation of the aversive brain stimulation and by the degree of preference shift away from an area associated with these stimuli. This indicates that rats are able to associate environmental cues with the consequences of central gray stimulation. These findings contrast with earlier findings demonstrating poor avoidance learning in response to central gray stimulation in a more complex active avoidance task (Cox, 1967). In that study, only 3 of 24 animals exceeded baseline values for spurious responses occurring during "dummy" control trials. Irisawa and Iwasaki (1986) also reported that active avoidance conditioning with central gray stimulation is difficult, and speculated that central gray stimulation may evoke primarily motor escape behaviors. The fearmotivated avoidance behavior obtained in the present study argues against this interpretation of the consequences of central gray stimulation.

It should be recalled that rats that fail to master a complex active avoidance task with central gray stimulation were able to master the same task in response to footshock (Cox, 1967). Consequently, it would appear that peripherally applied nociceptive stimulation provides more or different information than is generated by central gray stimulation and that this information is essential for avoidance learning in more difficult tasks. There are several possible differences between the events generated by peripheral footshock and central gray stimulation that may account for the performance difference obtained with central gray stimulation in simple and complex active avoidance tasks. One possibility is that central gray stimulation may be more emotionally disruptive and consequently may interfere with the acquisition of more complex active avoidance responses. Another possibility is that peripherally applied footshock provides spatial information regarding the source of nociceptive stimulation that may be needed for the directional responding required in the shuttlebox task. Such information may not be as critical in the simpler task employed in the present study, 
in which there were very salient environmental stimuli indicating the location of a safe area.

Finally, dorsal central gray stimulation may activate only a subset of systems involved in nociception and/or avoidance learning. Recent work has implicated a number of different brain areas in avoidance learning (Gabriel, Foster, Orona, Saltwick, \& Stanton, 1980; Gabriel, Gregg, Clancy, Kittrell, \& Dailey, 1986; Gabriel et al., 1983). Although dorsal central gray stimulation did yield active avoidance conditioning in a simple task in the present study, such stimulation may not engage neural systems that play an essential role in active avoidance learning in more complex tasks.

\section{REFERENCES}

Cox, V. C. (1967). Avoidance conditioning with central and peripheral aversive stimulation. Canadian Journal of Psychology, 21, 425-435.

Cox, V. C., \& VAlenstein, E. S. (1966). The effect of extensive mesencephalic central gray lesions on responses to reinforcing brain stimulation. Psychonomic Science, 4, 1-2.

Gabriel, M., Foster, K., Orona, E., Saltwick, S. E., \& StanTON, M. (1980). Neuronal activity of cingulate cortex, anteroventral thalamus, and hippocampal formation in discriminative conditioning: Encoding and extraction of the significance of conditioned stimuli. In J. M. Sprague \& A. N. Epstein (Eds.), Progress in psychobiology and physiological psychology (Vol. 9, pp. 126-231). New York: Academic Press.

Gabriel, M., GregG, B., Clancy, A., Kittrell M., \& Dailey, W. (1986). Brain stem reticular formation neuronal correlates of stimulus significance and behavior during discriminative avoidance conditioning in rabbits. Behavioral Neuroscience, 100, 171-184.

Gabriel, M., Lambert, R. W., Foster, K., Orona, E., SparenBORG, S., \& MAlorCa, R. R. (1983). Anterior thalamic lesions and neuronal activity in the cingulate and retrospenial cortices during discriminative avoidance behavior in rabbits. Behavioral Neuroscience, 97, 675-696.
IrISAWA, N., \& IwASAKI, T. (1986). Aversive-CS-specific alterations of evoked potentials in limbic and related areas of rats. Physiology \& Behavior, 37, 61-67.

Krieckhaus, E. E., Miller, N. E., \& Zimmerman, P. (1965). Reduction of freezing behavior and improvement of shock avoidance by d-amphetamine. Journal of Comparative \& Physiological Psychology, 60, 36-40.

Liebman, J. M., Mayer, D. J., \& Liebeskind, J. C. (1970). Mesencephalic central gray lesions and fear-motivated behavior in rats. Brain Research, 23, 353-370.

MEHLER, W. R. (1957). The mammalian "pain tract" in phylogeny. Anatomical Records, 127, 332.

Melzack, R., Stotler, W. A., \& Livingston, W. K. (1958). Effects of discrete brainstem lesions in cats on perception of noxious stimulation. Journal of Neurophysiology, 21, 353-367.

Nashold, B. S., Wilson, W. P., \& Slaughter, D. G. (1969). Sensation evoked by stimulation in the midbrain of man. Journal of Neurosurgery, 30, 14-24.

OLDS, M. E., \& OLDS, J. (1962). Approach-escape interactions in rat brain. American Journal of Physiology, 203, 803-810.

Phillips, A. G., Spyrakı, C., \& Fibiger, H. C. (1982). Conditioned place preference with amphetamine and opiates as reward stimuli: Attenuation by haloperidol. In B. G. Hoebel \& D. Novin (Eds.), The neural basis of feeding and reward (pp. 455-464). Brunswick, ME: Haer Institute for Electrophysiological Research.

STEIN, L. (1965). Facilitation of avoidance behavior by positive brain stimulation. Journal of Comparative \& Physiological Psychology, 60, 9-19.

VALENSTEIN, E. S. (1965). Independence of approach and escape reactions to electrical stimulation of the brain. Journal of Comparative \& Physiological Psychology, 60, 20-30.

Wolfle, T. L., MAYer, D. J., CARDER, B., \& Liebeskind, J. C. (1971). Motivational effects of electrical stimulation in the dorsal tegmennum of the rat. Physiology \& Behavior, 7, 569-574.

(Manuscript received August 25, 1986; revision accepted for publication January $21,1987$. 